\title{
Discussion of Foregoing Papers
}

\author{
H. M. POLLARD, M.D.*
}

The FIRST PORTION of this symposium abounds in marvelous material, particularly as related to the etiology of peptic ulcer. Dr. Dragstedt and Dr. Shay presented material on studies that shed valuable information on the development of duodenal as well as gastric ulcer.

Gastric Analysis: I agree completely with Dr. Klotz; I am a believer in gastric analysis. Its value depends much upon how you intend using the determinations in connection with the management of your ulcer patient. From knowledge of the degree of hyperacidity one can better rationalize with the patient their adherence to the program. The secretory patterns that may be characteristic of benign versus a malignant ulcer, I feel, are partially true. That a ulcerating nèoplasm in the stomach can be accompanied by a normal or even a high degree of free hydrochloric acid should be re-emphasized. The test is of greatest value when there is complete achlorhydria in the presence of an ulcerating lesion. There seems to exist in some individuals who normally secrete hydrochloric acid an achlorhydric phase. We recently evaluated an individual with an ulcerating lesion in the stomach who, on two studies, was achlorhydric; yet without our altering our technique in any way, the third test showed perfectly normal secretory activity, with free-acid secretion of 80 degrees. This, I do believe, results from an achlorhydric phase in that particular patient. Dr. Walter Palmer has referred to this possibility.

Dr. Sara Jordan has said that she can tell when her patients are smoking because their acidity, as demonstrated by gastric analysis, is much higher at such times.

The tubeless gastric analysis procedure has created considerable enthusiastic clinical interest. The test depends upon the liberation of a dye by contact with free hydrochloric acid (azure A combined with a resin). This dye is absorbed in the small intestine and excreted in the urine. The determination is performed on the urine, permitting a conclusion as to whether or not there is achlorhydria. It unfortunately gives only some index as to the height of the acidity. The procedure, depending upon what you desire of a

*Professor of Internal Medicine, University of Michigan, Ann Arbor, Mich.

Presented at the Peptic Ulcer Symposium, Louisiana State University, Nov. 1, 1958. 


\section{Pollard}

gastric analysis, therefore, has its limitations. When it is only desired to determine whether there is achlorhydria or not, or if the acidity is normal or slightly elevated, the test is actually informative. We have had infrequent instances of false positives in both directions. This is the type of procedure suited for a screening test. It is particularly of value when for one reason or another you cannot intubate a patient and in whom it is important to know the status of their gastric secretion.

Cytology: Dr. Arnold's excellent cytologic results and his estimate of their value in the differential diagnosis of gastric lesions are notable. We have had similar favorable experience. Gastric cytology has become one of the standard procedures in our institution, whereas only a few months ago, and certainly a few years ago, it was looked upon as experimental. We feel this study should always be done when there is any question about the identity of the lesion.

Gastroscopy: The endoscopy material of Dr. Nelson is excellent. A word of modification regarding the Fiberscope should be added. The information came from our unit that it was possible to see and to photograph through the Fiberscope. Whether or not we ever actually saw into the duodenum has not been authenticated. I do not believe that a duodenal lesion, therefore, has as yet been identified through the Fiberscope. We did, however, see through the Fiberscope the inside of the stomach and photograph it. The instrument has not been sufficiently perfected to permit a differential diagnosis of a gastric lesion. Much more has to be done in order to make it a usable instrument. In this respect, several technicians at the present time are working on its perfection, and possibly developments of importance will be soon reported.

In relation to selective biopsy of gastric lesions I agree with Dr. Nelson completely. This is a very difficult procedure and has not met with very wide acceptance. I was greatly impressed with his photography. The only factor that worries me is the lack of depth perception. Areas demonstrated as ulcers do not give the perspective of depth in the ulcer. The possibility of using a stereo camera to get proper depth depiction is suggested. In the polypoid lesion there was ease of identification.

Roentgenographic Interpretation: I particularly appreciated the limitations that Dr. Ruffin mentioned, and am in complete agreement with the necessity for clinical correlation. 